\title{
Hawking Radiation, the Unruh Effect and the Polarization of Electrons
}

\author{
Jon Leinaas \\ Institute of Physics, University of Oslo, Norway
}

\begin{abstract}
The polarization of electrons induced by quantum fluctuations of the vacuum field can be used as a robust radiation detector for the Unruh effect, where an idealized thermometer accelerated through vacuum registers a non-zero temperature, thereby providing a laboratory probe for Hawking radiation.
\end{abstract}

In standard quantum field theories, the vacuum state is the building block on which the full space of states of the system is based. It is the ground state of the Hamiltonian, and corresponds to "empty space" in the conventional understanding of space and matter. However, in quantum theories, vacuum is not completely free from structure. This structure can be understood in terms of "quantum fluctuations" of the fields fluctuations which are present even when there are no excitations (particles) present in the system. This vacuum activity is somewhat analogous to the zero point fluctuations existing in a material system at zero temperature, fluctuations which are, for example, responsible for helium remaining fluid even at the lowest possible temperatures.

Vacuum fluctuations can give rise to physical effects when the boundary conditions defining the vacuum are changed. Thus, if conducting plates are introduced into the electromagnetic vacuum, the fluctuations of the fields around the plates will be influenced by the plates, and this gives rise to forces between the plates (the well-known Casimir effect). However, even without referring to changing boundary conditions, there is a certain ambiguity in the definition of the vacuum state which comes from the fact that the Hamiltonian in relativistic theories is linked to the choice of space-time coordinates. Consequently, even when ordinary Minkowski-space quantum field theories are referred to non-inertial coordinates, the ordinary vacuum is no longer the ground state [1]. If a special choice of uniformly accelerated coordinates is made (Rindler coordinates) a new ground state can be found (Fig. 1). However, relative to this "accelerated vacuum", the ordinary Minkowski vacuum is not empty, but contains excitations (radiation).

Professor J.M. Leinaas is with the Institute of Physics, University of Oslo, Norway. He studied at the University and spent a year at the State University of New York at Stony Brook, USA before taking up Fellowships at NORDITA, Oslo $(1978 / 80)$ and CERN, Geneva (1981/82).

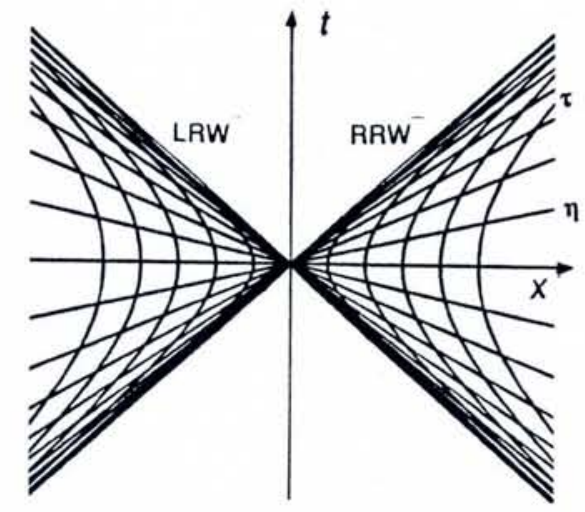

Fig. 1 - Accelerated Rindler coordinates ( $\tau)$. Ordinary Minkowski space is hot with respect to the corresponding Hamiltonian, defined in either the "right Rindler wedge" (RRW) or in the "left Rindler wedge" (LRW). A uniformly accelerated point detector, following a path with $\eta$ fixed is heated due to interactions with vacuum fluctuations.

\section{Hawking Radiation}

The physical vacuum is influenced not only by physical boundaries, but also by the presence of external fields. Thus, the vacuum fluctuations of charged fields will be influenced by electric fields, and if these become sufficiently strong, the vacuum is no longer stable: the strong electromagnetic field gives rise to spontaneous pair creation. Strong gravitational fields may also give rise to similar vacuum effects. As shown by Hawking [2], the vacuum fields around a black hole are unstable and spontaneously emit energy quanta.
An interesting feature of this theoretical effect is that the radiation is thermal, with the temperature given by

$$
k T_{H}=\frac{\hbar c^{3}}{8 \pi G M}
$$

where $k$ is Boltzman's constant, $G$ is Newton's gravitational constant and $M$ is the mass of the black hole.

Hawking derived this result by considering the continuation of field modes through a collapsing massive body. However, it seems clear that such an effect is also present for an eternal black hole, and the presence of radiation is then related to the problem of defining a stationary vacuum state in the gravitational field of the black hole.

\section{Unruh Effect}

Formally, there is a close resemblance between the Hawking effect and the presence of radiation quanta in an ordinary Minkowski vacuum when the latter is referred to the accelerated Rindler coordinates. This resemblance is demonstrated in a clear way by what is often referred to as the Unruh effect. According to Unruh [3], an idealized detector which is uniformly accelerated through Minkowski vacuum will detect radiation with a thermal spectrum. The temperature formula has a form closely related to that for Hawking radiation,

$$
k T_{u}=\frac{\hbar a}{2 \pi c}
$$

with $a$ as the acceleration of the detec-
From the left, J.M. Leinaas, H. Wind, $H$. Overaas and John Bell at CERN in August 1987.

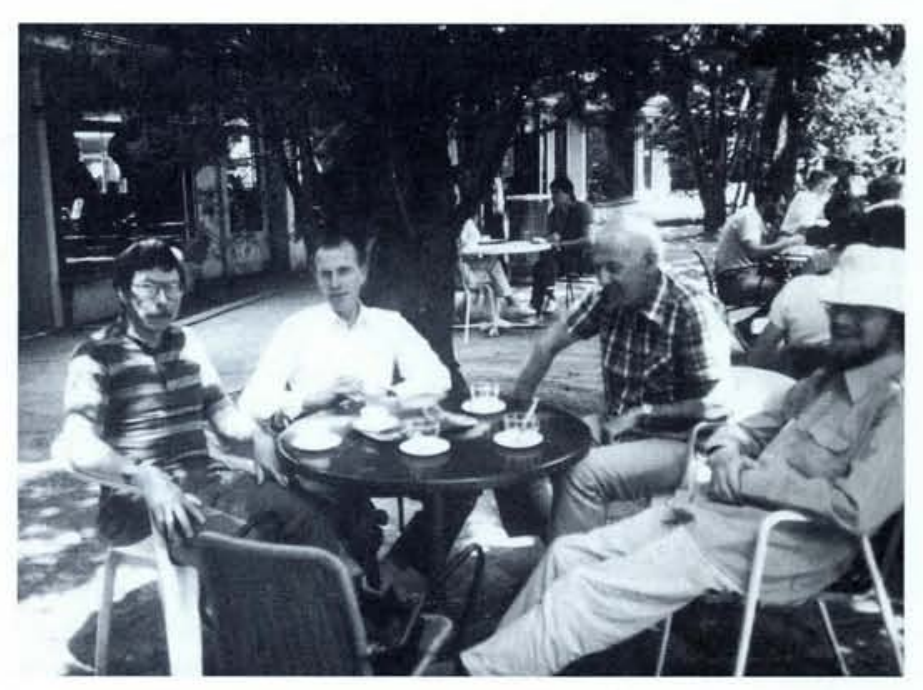


tor (as measured in an inertial rest frame). In the same way as the Hawking temperature is extremely small for black holes of astronomical size, the Unruh temperature is extremely small for "normal" accelerations. By inserting numbers into the temperature formula one realizes that even a modest temperature of $1 \mathrm{~K}$ requires an acceleration of $a=$ $2.4 \times 10^{20} \mathrm{~m} \mathrm{~s}^{-2}$ : an extremely robust detector is clearly needed to withstand such accelerations. One may therefore wonder if this has to remain a purely theoretical effect, or whether the vacuum temperature may be seen in real experiments.

\section{Polarized Electrons}

A few years ago, as a Fellow at CERN, I discussed these problems with John Bell and we became interested in examining matters a little further. We were led to consider elementary particles as possible radiation detectors, since other physical systems hardly could be sufficiently robust at the required accelerations. As suggested by John Bell, one might ask whether the temperature effect could be related to a polarization effect which was known to exist for electrons circulating in a magnetic field. It had already been established that electrons in a storage ring polarized spontaneously, but not completely [4]: the maximum polarization had been found to be $P=0.92$. Could the departure from full polarization be interpreted as due to heating of the electrons caused by their acceleration?

As a result of our investigation, the answer to this question was a qualified affirmative. The effect was indeed of the same type, but there were complications owing to the fact that the electrons were circulating instead of accelerating linearly; Thomas precession was another complicating factor. I will briefly outline how the polarization effect could be related to the Unruh effect and also point to the complications (refer to [page 72, 7] for more details).

\section{Polarization and the Unruh Effect}

Electrons in a magnetic field $B$ can naively be thought of as a thermometer in the following way: assume for the moment that a particle's position is unimportant and that only the spin degree of freedom is of interest. The spin Hamiltonian has the form

$$
H=\frac{1}{2} \hbar \vec{\omega} \cdot \vec{\sigma}
$$

with $\vec{\omega}$ as the Pauli spin matrices and $\vec{\sigma}$ as the vector $(\mathrm{eg} / 2 \mathrm{mc}) \boldsymbol{B}$ where $g$ is the gyromagnetic factor, which is close to 2 for electrons, and $-e$ the electron
Fig. 2 - A simplified picture of electrons in a magnetic field, where only the spin interaction with the field is considered. At zero temperature, all the particles sit in the ground state, with spin antiparallel to the magnetic field. At non-zero temperatures, some particles are excited to the upper energy level, with spin parallel to the magnetic field.
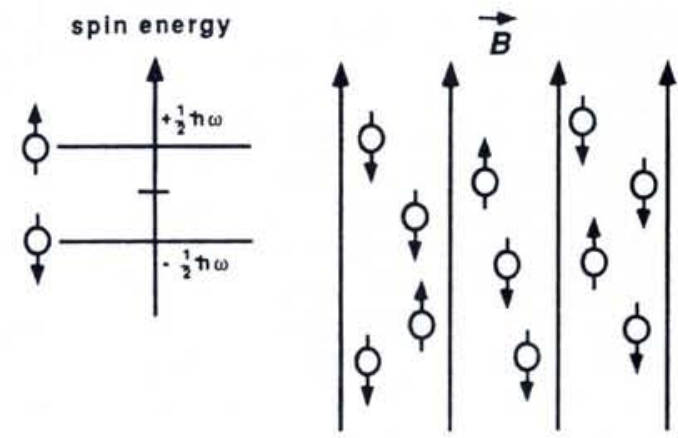

Fig. 3 - Polarization curves for electrons circulating in a magnetic field as functions of the gyromagnetic factor $\mathrm{g}$. $A$ is the curve found using a naive application of Unruh's temperature formula: $B$ is the curve for a more detailed analysis.

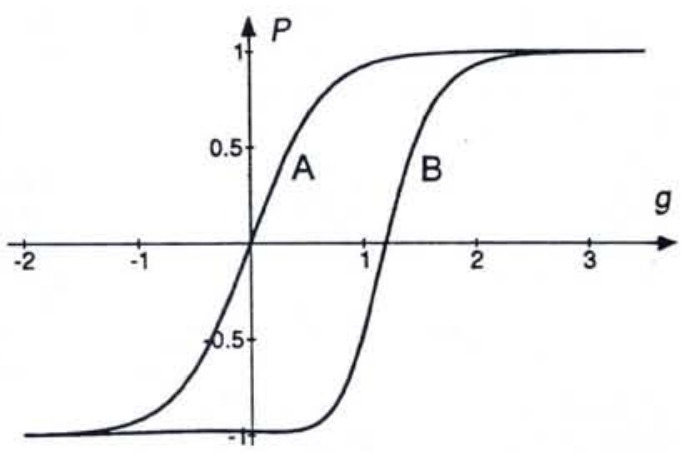

charge. An ensemble of such electrons at zero temperature will be fully polarized in the direction opposite to that of the magnetic field since they will all sit in the ground state (Fig. 2). However, for a non-zero temperature $T$ there is a certain probability for excitation to the upper energy level. The ratio $R$ between the occupation of the corresponding spin levels is then

$$
R=\exp \left(-\frac{e \hbar g B / 2 m c}{k T}\right)
$$

and the polarization is

$$
P=\frac{1-R}{1+R}=\tanh \left(\frac{e \hbar g B / 4 m c}{k T}\right)
$$

If this naive picture is applied to electrons circulating in a magnetic field and Unruh's temperature formula is used, one finds

$$
P=\tanh \left(\frac{e \hbar g B^{\prime} / 4 m c}{e \hbar E^{\prime} / 2 \pi m c}\right)=\tanh \left(\frac{\pi g}{2 \beta}\right)
$$

where $B^{\prime}=\gamma B$ and $E^{\prime}=\beta \gamma B$ are the magnetic and electric fields in the rest frame of the electrons, $\beta=v / c$ close to 1 for highly relativistic electrons and $\gamma=\left(1-\beta^{2}\right)^{-1 / 2}$. For $g=2$ this gives $P$ $=0.996$ as compared with $P=0.924$ found in earlier QED calculations. The naive picture is clearly too simple to give the correct result. However, it is interesting to compare the results for $g$ not equal to 2. As shown in Fig. 3, the main difference between the function $P(g)$ given above and that of the more complete QED calculation [5] is a shift of the curve along the $g$-axis. Such a shift is in fact not totally unexpected. Taking account of the Thomas precession of the electron in the definition of the spin the $g$-axis, but with 2 units rather than with about 1.2 which seems to be more correct.

Another indication that the main part of the complication relates to a spin effect is seen if one considers a modified problem where the electromagnetic field is replaced by a scalar radiation field. If a two-level system is coupled linearly to this field, and the occupation ratio $R$ is calculated as a function of the energy splitting for circular motion, then the deviation from the result found using the Unruh formula is very small [page 72, 7].

\section{Exact Analysis}

In a more serious treatment of the electrons circulating in a storage ring, we introduced the Hamiltonian defined in an accelerating coordinate frame, moving along the classical path of a circulating electron. With rotationally invariant external fields, this Hamiltonian is time-independent, and the situation is stationary as seen from the moving electron. The Hamiltonian has the form (1) as before, but with a somewhat different form for the vector $\vec{\omega}$, which now depends both on the classical external fields and the quantum radiation field, as well as on the position vector and velocity of the electron-variables describing fluctuations around the classical path.

The classical fields define the energy levels of the spin Hamiltonian, and the quantum fluctuations determine the Hamiltonian would shift the curve along 
excitation probabilities. In our approach, these excitations come about in two ways: the first is through the direct coupling of the spin vector to the quantized electromagnetic field; the other is through fluctuations in the particle orbit. It is the vertical fluctuations (in the direction of the magnetic field) which induce spin transitions. Through the Heisenberg equations of motion, these fluctuations in turn can be related to the quantum fluctuations in the electromagnetic fields. The field fluctuations build up the fluctuations in the orbit and radiation damping prevents them from growing indefinitely.

The part of the fluctuations in the vector $\vec{\omega}$, which is responsible for the spin excitations, can then be written in the simple form

$$
\delta \vec{\omega}=\frac{e}{2 m c}\left[g B_{\mathrm{q}}^{\prime}+\left(2 e_{\mathrm{r}}+f(\mathrm{~g})\right) E_{\mathrm{qz}}^{\prime}\right]
$$

where $B_{\mathrm{q}}^{\prime}$ and $E_{\mathrm{qz}}^{\prime}$ are the free quantum fields in the rest frame of the electron and $e_{r}$ is a unit vector in the radial direction. The resonance factor $f(g)$ is proportional to $g$-2: being a function of $\gamma$ it is essentially equal to 0 except in a narrow interval where there is resonance between the vertical fluctuations and the spin motion. The transition probabilities between the spin levels now are determined by correlation functions of along the classical orbit. For $\boldsymbol{f}(g)=0$, the result found in this way for the transverse polarization of the electrons agrees with the earlier result [5].

An interesting additional observation concerns the resonance factor $f(g)$. In our approach one sees that the resonance does not have a purely depolarizing effect. As a function of $\gamma$, the polarization close to the point of resonance initially decreases, from 0.92 to -0.17 , but then increases to 0.99 before finally decreasing again to 0.92 . It is thus possible, at least in principle, to exceed the "maximum" value 0.92 close to the resonance.

\section{Conclusions}

Returning to the question of the relation between the polarization effect and the Unruh effect one sees, as already stated, that they are of a similar nature. Transitions between spin levels are caused by quantum fluctuations of the vacuum fields along the electron orbit, in the same way as field fluctuations induce transitions in a linearly accelerated detector. In this sense, both the electrons and the detector are heated by the accelerating motion through a vacuum.

However, there is also an essential difference between the two cases. For uniform linear acceleration, the excitation spectrum has a universal thermal character. This is not the case for the "circular Unruh effect" where the excitation spectrum depends on characteristics of the detector. Using circulating electrons, the effects of Thomas precession are important and fluctuations in the orbit cannot be neglected.

\section{ADDITIONAL REFERENCES}

[1] Fulling S.A., Phys. Rev. D 7 (1973) 2850. [2] Hawking S., Nature 248 (1974) 30; Commun. Math. Phys. 43 (1975) 199.

[3] Unruh W.G., Phys. Rev. D 14 (1976) 870.

[4] Sokolov A.A. and Ternov I.M., Dokl. Akad. Nauk. SSR 153 (1963) 1052.

[5] Derbenev Ya.S. and Kondratenko A.M. Zh. Exp. Teor. Fiz. 64 (1973) 1918; Jackson J.D., Rev. Mod. Phys. 48 (1976) 417.

\section{John Bell Symposium}

A Symposium on Quantum Physics, in memory of John Stewart Bell who died on 1 October 1990, will be held at CERN on 2-3 May. The programme includes talks by $A$. Aspect, H. Rauch, A.J. Leggett, K. Gottfried, A. Shimony, G.C. Ghirardi, J. Leinaas and R. Jackiw. Further information: Jeanne Rostant, Theory Division, CERN, CH-1211 Genève 23 (Tel./Fax: +41 (22) $7674222 / 39$ 14; Bitnet: rostant@cernvm.cern.ch).

\section{World Lab Fosters Third World Excellence}

Establishing a "new equilibrium" in the distribution of knowledge between east and west, north and south can provide a "new way out" for undeveloped countries by allowing the creation of scientifically based cultures which have the capacity to generate wealth from limited material resources. But one has to act directly because if one "applies European logic", this process would never have started even in our "corner of the planet". Action must also span all disciplines since "only a strong civilization creates science". Most important of all, experience in creating the great laboratories of Europe implies that solutions will mostly spring from initiatives by individuals.

These remarks by Professor Antonino Zichichi help one understand the principles which guide the International Centre for Science Culture (ICSC), better known as the World Laboratory. Founded by Professor Zichichi, its President, in 1986, the World Lab works to extend the Erice heritage in improving the training of, and opportunities for, scientists based in poorer countries. Erice is the home of the Ettore Majorana Centre for Scientific Culture that celebrated its 25th Anniversary last year with the first award of the
Science for Peace Prize (see Europhysics News 21 (1990) 219). Housed in two converted monasteries and a former convent in the famous medieval hill-top town, the Centre has hosted some 100 schools and 480 courses attended by 44000 participants from 103 countries, a great demonstration of "international collaboration without ideological position". Erice also gives its name to the world renowned Statement written by P.A.M. Dirac, P.L. Kapitza and A. Zichichi whose 10000 signatories declare themselves morally bound to work purely in the interests of science - a "new rôle for science".

\section{World Laboratory}

The World Lab builds upon the Erice principles through collaborative projects in science and technology with existing institutions. The Lab's operating budget comes largely from Italy, the USSR and China, but this does not imply that it is just another funding agency. Each beneficiary country in fact handles local projects, thus requiring only a very small administrative team at the headquarters in Lausanne, Switzerland.

The Erice experience also provided a wealth of essential experience and con-

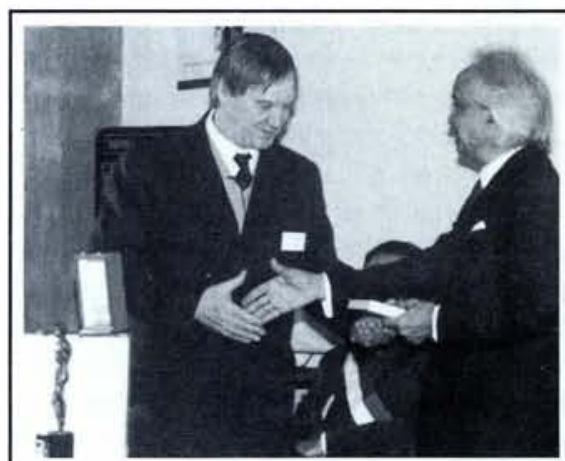

Professor S.P. Kapitza (left) receiving on behalf of his father Academician P.L. Kapitza the first Scienza per la Pace Prize from Professor A. Zichichi last November in Erice, Sicily.

The other winners were P.A.M. Dirac and A.D. Sacharov, E. Teller and V.F. Weisskopf.

tacts so that distinguished colleagues can help identify unique projects. The result is that Professor T.D. Lee of Columbia University was instrumental in setting up in 1986, Beijing's Center for Advanced Science and Technology of which he is the Director. The Center is a branch laboratory established with governmental agreement that ensures independence and effectiveness in running projects in China. It employs 50 scientists and 30 students and, aside from research in high energy 